\title{
ARTIGOS
}

\section{COMPARAÇÃO DO IMPACTO AMBIENTAL DE CAPA DE LAPTOP UTILIZANDO A \\ AVALIAÇÃO DO CICLO DE VIDA}

\section{ENVIRONMENTAL IMPACT COMPARISON OF LAPTOP SLEEVE CASE BAGS USING LIFE CYCLE ASSESSMENT}

\author{
LUISA PEREIRA BASILE | UFPR \\ MARCELL MARIANO CORRÊA MACENO, DR. | UFPR \\ ADRIANA DE PAULA LACERDA SANTOS, DRA. |UFPR
}

\begin{abstract}
RESUMO
O presente artigo objetivou comparar o impacto ambiental de uma capa de laptop, produzida parcialmente com materiais reciclados, por integrantes de projetos sociais da Fundação de Ação Social da Prefeitura Municipal de Curitiba (FAS), com outras duas capas de laptop comerciais (corino e neoprene), através da utilização da metodologia de avaliação do ciclo de vida de produtos. Para o desenvolvimento da ACV foram adotadas as recomendações das normas ISO 14040. Os dados foram trabalhados no software SimaPro v. 8.0.5, com modelagem em relação aos impactos ambientais. Foi utilizada a base de dados do inventário Ecoinvent v.3, para definição do inventário do ciclo de vida do produto, e o método de avaliação IMPACT 2002+ para identificação dos impactos ambientais. Após aplicação da metodologia ACV concluiu-se que as capas de laptop que não utilizavam matéria-prima reciclada geraram menor impacto ambiental.
\end{abstract}

PALAVRAS CHAVE: Impacto Ambiental; Avaliação do Ciclo de Vida; Sustentabilidade

\begin{abstract}
The present article aimed to compare the environmental impact of a laptop cover, which was partially produced with materials reused by members of social projects of the Curitiba Social Action Foundation (FAS), with two commercial laptop covers (artificial leather and neoprene), through the use life cycle assessment. The ISO 14040 standards were adopted for the LCA development. The data were analysed on the software SimaPro v. 8.0.5, modeling in relation to environmental impacts. The Ecoinvent v.3 inventory database was used to define the product life cycle inventory and the IMPACT 2002+ assessment method for identifying environmental impacts. After applying the LCA methodology it was concluded that laptop covers that did not use reused material generated less environmental impact.
\end{abstract}

KEY WORDS: Environmental Impact; Life Cycle Assessment; Sustainability 


\section{INTRODUÇÃO}

O aumento de discussões sobre a gestão ambiental no século XXI tornou indispensável a toda corporação o debate sobre sustentabilidade. As esferas econômica, ambiental e social, também chamadas tripple bottom line, passaram a ser consideradas como condição para se atingir uma produção sustentável (GMELIN E SEURING, 2014).

As empresas necessitam crescer, se adaptar ao mercado consumidor, atender as necessidades e satisfação dos clientes. Ou seja, elas passaram a ter que considerar como estratégia de mercado, e também de sobrevivência, as exigências apresentadas por consumidores, empresas parceiras ambientalmente conscientes e governo (CARVALHO et al., 2014), bem como de legislações mais rigorosas, padrões internacionais e a própria competição de mercado. Neste contexto, além da agilidade, produtividade e alta qualidade, a preocupação com o meio ambiente passou a ser fator importante para o sucesso de uma organização.

Assim, de acordo com Herzog (2004), produtos que visam a sustentabilidade são mais propensos a ter mais saída no mercado consumidor, pelos investidores e pelos governos. Portanto, a sustentabilidade hoje, é uma meta a ser buscada, dado que, a preocupação em atingir e demonstrar um comportamento ambientalmente adequado, reflete na imagem do fabricante ante a sociedade, aumentando a lucratividade e competitividade dele em seu mercado de atuação (CENTRO SEBRAE DE SUSTENTABILIDADE, 2013).

Nesse contexto, a Avaliação do Ciclo de Vida (ACV) passa a ser uma ferramenta essencial, auxiliando a análise, comparação e a tomada de decisão sobre produtos de modo a avaliar a melhoria ou não de seu desempenho ambiental (GMELIN E SEURING, 2014).

Nos casos de novos produtos advindos de reuso, reaproveitamento ou reciclagem de materiais, como os produtos fabricados e comercializados por associações cooperativas, também conhecidas por Arranjos Produtivos Locais (APLs), espera-se de forma concreta a diminuição de resíduos e a prevenção a extração de recursos naturais (GUTBERLET, 2015), possivelmente reduzindo o impacto ambiental deste tipo de produtos.

Desta forma, este artigo objetivou comparar o produto capa de laptop fabricado por uma APL ligada a Fundação de Ação Social de Curitiba com outros dois modelos industrializados presentes no mercado.

\section{REVISÃO BIBLIOGRÁFICA}

Como forma de esclarecer e detalhar assuntos de interesse para o entendimento deste artigo, são apresentados nos tópicos 2.1, 2.2 e 2.3 os temas de Sustentabilidade, Processo de Desenvolvimento de Produto (PDP) e Avaliação do Ciclo de Vida (ACV), respectivamente.

\subsection{Sustentabilidade}

A revolução industrial e a revolução tecnológica, ocorridas durante os últimos três séculos, permitiram o surgimento de novas técnicas produtivas, necessárias para aumentar a capacidade de produção num mundo em crescente crescimento demográfico. Entretanto, esse aumento produtivo trouxe consigo efeitos colaterais, que provocaram questionamentos sobre a capacidade de sobrevivência humana (OLIVEIRA ET AL, 2012).

Esses questionamentos deram origem a diversas correntes de pensamento, assim como estudos e pesquisas, que buscavam chegar a um modelo de desenvolvimento que permitisse a interação das necessidades humanas com o meio ambiente e com outros seres vivos.

Dessas discussões, surgiu o termo "sustentabilidade", que foi apresentado oficialmente na reunião da Comissão Mundial sobre Meio Ambiente e Desenvolvimento (CMMAD), da Organização das Nações Unidas (ONU), ocorrida em 1988. Sustentabilidade foi definida como "[...] a capacidade de satisfazer as necessidades do presente sem comprometer a capacidade das gerações futuras de satisfazerem suas próprias necessidades" (CMMAD, 1988, p. 9).

Conforme coloca Mikhailova (2004, p. 1), “É provável que, nos anos recentes, nenhum conceito tenha sido citado tantas vezes, discutido e empregado em tantas pesquisas, como o conceito de desenvolvimento sustentável e de sustentabilidade".

Dentro desta discussão, entende-se que a sustentabilidade é composta de três dimensões, que se relacionam: econômica, ambiental e social. Essas dimensões são também conhecidas como tripple bottom line (OLIVEIRA et al., 2012); conceito que teve origem em estudo realizado por Elkington (1994) sendo também conhecido como 3P (People, Planet e Profit). Em português, 3P é traduzido como PPL (Pessoas, Planeta e Lucro). Dessa maneira, uma produção sustentável seria aquela que apresenta as três dimensões: 1) econômica, que implica na criação de empreendimentos viáveis e atraentes para os investidores; 2 ) ambiental, que exige que os processos tenham interação com o meio ambiente, sem causar danos permanentes e 3) social, que supõe o estabelecimento de ações justas para trabalhadores, parceiros e sociedade. 
Na atualidade, o debate sobre aquecimento global vem colocando o tema da sustentabilidade, principalmente ambiental, como prioridade das grandes empresas. Conforme colocam Arruda e Quelhas (2010), isso tem feito com que lideranças mundiais venham se mobilizando, na tentativa de alinhar as práticas empresariais com valores socioambientais mais justos. Arruda e Quelhas (2010) citam empresas que apresentam na internet relatórios e políticas de sustentabilidade baseadas no mapeamento/gerenciamento de riscos ambientais; bom relacionamento com clientes, fornecedores e comunidade e incorporação de processos de inovação tecnológica voltados para uma produção eficiente. Assim sendo, muitas empresas para manterem a sustentabilidade ambiental já adotam medidas no projeto do produto. De acordo com Assis (2009), é importante aliar a sustentabilidade a Gestão de Produtos, principalmente no projeto do produto, evitando características que possam gerar riscos ao meio ambiente e não acordo com normas ambientais.

\subsection{Processo de Desenvolvimento de Produto} (PDP)

De acordo com Freitas et al. (2014), as inovações dentro das empresas podem ser alcançadas a partir do Processo de Desenvolvimento de Produtos (PDP), que seria um processo de negócio, ou seja, seu foco é resultar em produtos que tenham valor para os clientes da empresa. O PDP deve ser estruturado segundo um modelo de referência, que integre atividades, recursos, informações e boas práticas e é classifica do em três etapas: Pré-desenvolvimento, Desenvolvimento e Pós-desenvolvimento.

De acordo com Faria et al. (2008), o Prédesenvolvimento, também chamado de planejamento do produto, é a fase na qual é definido o produto a ser desenvolvido; ou seja, o escopo do projeto, além da avaliação econômica, avaliações de capacidade de risco, definição de indicadores para monitoramento e definição de planos de negócio do projeto. A fase de Desenvolvimento contém diversas etapas, como a Projeto Informacional, Projeto Conceitual, Projeto Preliminar e Projeto Detalhado, a fim de estruturar totalmente o produto e poder enfim realizar o seu lançamento no mercado. Na fase de Pós-desenvolvimento é feito o planejamento de como o produto será acompanhado e retirado do mercado. Conforme Rosenfeld et al. (2006) o Desenvolvimento de produto também envolve o acompanhamento do mesmo após o lançamento, bem como o planejamento da descontinuidade do produto no mercado, incorporando estes conceitos na especificação do projeto, atendendo assim, todas as necessidades do produto ao longo do seu ciclo de vida (ROSENFELD et al., 2006).

Acompanhando a discussão sobre sustentabilidade, Manzini e Vezzoli (2005) afirmam que o PDP deveria ser repensado, para que as dimensões da sustentabilidade possam ser obedecidas desde a concepção até o fim da vida do produto, tendo em vista que qualquer produção tende a provocar algum impacto ambiental.

\subsection{Avaliação do Ciclo de Vida (ACV)}

De acordo com Jensen e Rernrnen (2006), adotar o conceito de Avaliação do Ciclo de Vida na produção significa identificar os impactos que ocorrem desde a concepção até o fim da vida de um produto ou material. A Avaliação do Ciclo de Vida (ACV) ou Life Cycle Assessment (LCA) é uma ferramenta que visa abordar os aspectos ambientais de um produto e os impactos potenciais ao longo do ciclo de vida (UNEP/SETAC, 2009).

A ACV é definida como sendo a "compilação e avaliação das entradas, saídas e impactos ambientais potenciais de um sistema de produto ao longo do seu ciclo de vida" (ABNT, 2009, p. 2) e é regida pela série de Normas ISO 14040 .

A ACV é dividida em quatro fases, sendo elas: definição de objetivo e escopo, análise de inventário, avaliação de impactos e interpretação de resultados (Figura 1).

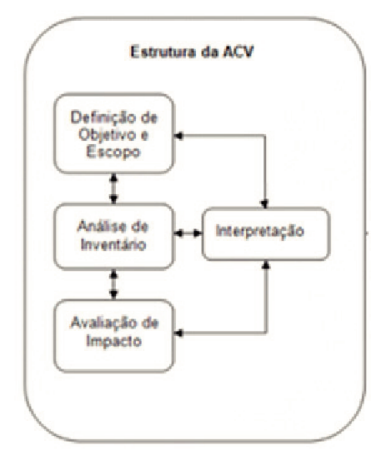

Figura 1: Fases da Avaliação do Ciclo de Vida. Fonte: ABNT (2009).

Na fase de definição do objetivo e escopo, primeiramente se faz a definição do objetivo do estudo, o qual deve conter a aplicação da ACV, as razões pretendidas, o público-alvo ao qual se destina o estudo e a intenção de divulgação pública dos resultados. Após definir o objetivo, deve-se estabelecer o escopo, que consiste nos limites em que o estudo é válido, dentre os quais se destacam: a função, a unidade funcional, o fluxo de referência, as fronteiras e o sistema do produto, informações relativas aos dados (como alocação, requisitos dos dados) e à avaliação 
de impacto de ciclo de vida (como a seleção de categorias de impacto). No escopo é interessante ressaltar que a metodologia ACV possui diferentes abordagens e nomenclaturas que diferem de acordo com os processos estudados.

A função serve para determinar qual o principal objetivo do produto analisado e a unidade funcional é a base de referência do estudo, à qual todas as entradas e saídas do sistema estarão relacionadas. O fluxo de referência corresponde a medida das saídas dos processos do sistema, necessária para o cumprimento da função expressa na unidade funcional (ISO 14040, 2009). Além disso, tem-se nomenclaturas para sistemas de produto, definidas pela ISO/TS 14048/2002, conforme segue (ABNT, 2002):

- Berço ao portão (Cradle-to-gate): o processo começa com a extração de recursos, que pode incluir alguns serviços de manufatura e operações, mas exclui todas as fases subsequentes;

- Berço ao túmulo (Cradle-to-grave): o processo começa com a extração de recursos e vai até o descarte final do produto;

- Portão ao portão (Gate-to-gate): o processo onde todas as etapas de produção ocorrem dentro de um local específico. O local pode ser geograficamente especificado, ou, no caso de dados genéricos, a especificação geográfica pode ser mais geral. Processos fora dos portões do local definido não estão incluídos;

- Portão ao túmulo (Gate-to-grave): o processo inclui a distribuição, uso e disposição final do produto.

Posteriormente, em uma segunda fase, é feita a Análise de Inventário do Ciclo de Vida. Essa fase engloba a coleta de dados em cada processo elementar e posterior processamento dos dados ao longo do ciclo de vida, resultando no Inventário do ciclo de vida (ICV). O ICV tem como resultado a somatória dos fluxos elementares, sendo esses fluxos provenientes do ou destinados ao meio ambiente.

Na sequência é realizada a fase da Avaliação de Impacto do Ciclo de Vida (AICV), a qual consiste em relacionar os resultados dos ICVs às categorias de impacto (toxicidade humana, uso da terra, mudanças climáticas, entre outros), abordadas por um determinado método de caracterização de impactos (ADISSI et al., 2013). O cálculo realizado pelo método de caracterização consiste em multiplicar as quantidades das espécies levantadas no inventário pelos seus respectivos fatores de caracterização de impacto, obtendo-se os valores dos indicadores de categoria de impacto. Desta forma, o resultado pode ser expresso na forma de indicadores de categoria, ou midpoints, categorias de danos, ou endpoints, e também na forma de Ecoponto (Pt), conforme o método de caracterização escolhido para a obtenção da AICV (MACENO, 2015).

Por fim, a fase de Interpretação está presente em todas as fases, entretanto, em uma ultima etapa da ACV, verificam-se os resultados em relação ao escopo. Um dos itens mais comuns dessa fase consiste em avaliar a contribuição, seja dos processos, seja dos fluxos elementares, no resultado final. (ADISSI et al., 2013).

\section{METODOLOGIA}

A pesquisa desenvolvida utilizou-se da metodologia ACV (Avaliação do Ciclo de Vida), baseada na ISO 14040 (ABNT, 2009). Para isso, foram necessárias as definições das seguintes etapas: Definição de Objetivo (compreendendo a aplicação, razão e público alvo) e Escopo (compreendendo a Função, Unidade Funcional, Fluxo de Referência, Sistema e Limite de Sistema, e Método de caracterização), o Inventário do Ciclo de Vida (compreendendo os dados levantados e coletados de massa e energia dos produtos), a Avaliação do Impacto do Ciclo de Vida (compreendendo a simulação do ICV através do método de caracterização definido), e a Interpretação dos resultados. O detalhamento destas etapas segue tópicos 3.1 à 3.3.

\subsection{Definição de Objetivo e Escopo}

Para Definição do objetivo e escopo da pesquisa (Fase 1) levou-se em consideração a motivação e no que a pesquisa pode ser aplicada, assim como seu público alvo.

Neste contexto, o objetivo da Avaliação do Ciclo de Vida da capa de laptop foi comparar a capa de laptop artesanal produzida por um APL da FAS com outros dois modelos de capa comercial de laptop (Corino e Neoprene) em relação ao impacto ambiental potencial delas. A Figura 2 apresenta as fotos das 3 capas de laptop analisadas, como forma de exemplificar os produtos.

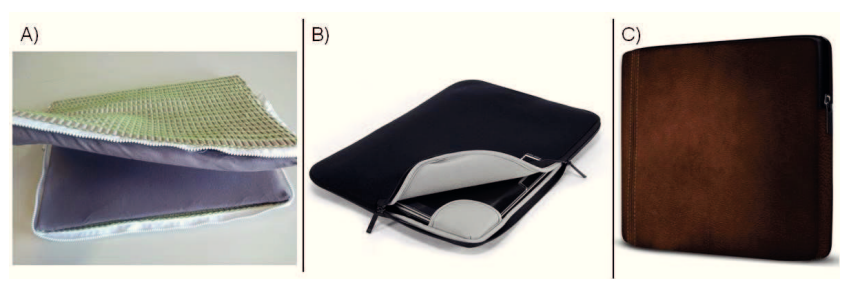

Figura 2: Fotos representativas das capas de laptop. A)Capa artesanal, B)Capa de neoprene, C)Capa de corino.

Fonte: A) os Autores, B) 4FILESTORE (2018), C) CASENOTEBOOK (2018)

O público alvo desta pesquisa foram os fabricantes da capa de laptop envolvidos nos Arranjos Produtivos Locais, apoiados pela FAS, e também do mercado em geral, além 
de interessados na metodologia ACV, estudantes e profissionais do meio acadêmico.

Em relação ao escopo do estudo, foram considerados o sistema do produto e limites, suas funções, unidade funcional e fluxo de referência, o método de avaliação de impacto e as limitações que foram considerados para a síntese do escopo.

Assim, o sistema do produto considerado foi a própria capa de laptop, sendo classificado este sistema como do berço-ao-portão, dado que foram analisados dados de materiais e energia desde a extração da matéria-prima até a produção das capas. Os materiais componentes das capas de laptop, e também os processos utilizados na fabricação delas, foram levantados, e as informações das etapas anteriores do ciclo de vida destes materiais e processos foram obtidos de bases de dados. Estas informações estão mais detalhadas no tópico 3.2.

A função, unidade funcional e fluxo de referência definidas para os produtos segue Quadro 1.

Em relação ao método de caracterização dos impactos ambientais potenciais, foi utilizado o método IMPACT2002+, sendo considerado um dos métodos mais utilizados, conforme aponta estudo de Carvalho et al. (2014).

Por fim, o processo de simulação da ACV foi realizada através da utilização do software SIMAPro 8.0.5. Este software é considerado o software mais popular e mais utilizado para avaliação do ciclo de vida de produtos.

\begin{tabular}{l|l}
\hline Função & Proteger o laptop \\
\hline $\begin{array}{l}\text { Unidade } \\
\text { Funcional }\end{array}$ & $\begin{array}{l}\text { Proteger } 1 \text { laptop de 17" contra } \\
\text { danos mecânicos }\end{array}$ \\
\hline $\begin{array}{l}\text { Fluxo de } \\
\text { Referência }\end{array}$ & 1 capa de laptop \\
\hline
\end{tabular}

Quadro 1: Definição da função, unidade funcional e fluxo de referência da ACV.

Fonte: Elaborado pelos autores.

Fonte: os Autores

\subsection{Inventário do Ciclo de Vida}

Na fase 2, ICV, foi realizada uma coleta de dados, identificando-se as matérias-primas da capa de laptop artesanal produzida pela FAS e também pesquisa de materiais utilizados nas capas produzidas industrialmente e suas respectivas quantidades. Os materiais da capa de laptop produzida na APL considerados pela pesquisa foram apenas aqueles não reaproveitados, visto ter sido considerada uma abordagem em que os materiais reaproveitados não carregariam o impacto de seu processo anterior ao reaproveitamento, também chama de abordagem Cut off. A árvore do produto da capa de laptop artesanal foi construída de modo a exemplificar as partes do produto, sendo apresentada na Figura 3.

Já a capa de Corino apresentou como partes de produto para sua composição o Zíper, Espuma e Fio de costura. Por fim, a capa de Neoprene apresentou como partes de produto para sua composição o Zíper e o Fio de costura.

Para a medição da quantidade de cada material da capa produzida pela FAS foi utilizada a balança de precisão "Mettler Toledo", modelo AL 204. Para as capas industrializadas, as informações sobre seus materiais foram encontradas em sites dos fabricantes e também em especificações técnicas dos produtos, sendo que foram consideradas duas capas para comparação, uma feita de corino e outra de neoprene.

$\mathrm{Na}$ sequência, efetuou-se a busca desses materiais na biblioteca de inventário do software SimaPro, versão 8.0.5. A base de dados de inventário priorizada dentro do Software foi a Ecoinvent v.3.

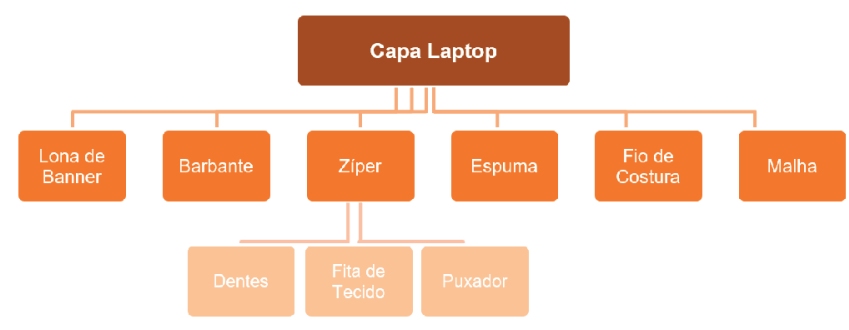

Figura 3: Árvore do produto capa artesanal. Fonte: 0s Autores

\subsection{Avaliação do Impacto do Ciclo de Vida e Interpretação}

Para a AICV (Fase 3), foi realizada no software SimaPro uma comparação entre os três modelos de capa, utilizando o método IMPACT 2002+. O software gerou gráficos, que serviram como base para a posterior análise.

Em cada uma das fases apresentadas foi realizada uma Interpretação dos dados (Fase 4), visando revisá-los, comprovando sua sensibilidade e consistência.

O método ACV aplicado pode ser considerado simplificado, uma vez que a pesquisa foi realizada de forma parcial, tendo em vista as limitações dos estágios do ciclo de vida, decorrentes das restrições da fonte de dados e tempo necessário. Entretanto, entende-se que a simplificação efetuada não comprometeu o alcance dos objetivos propostos. 


\section{RESULTADOS E DISCUSSÃO}

Este estudo teve como interesse comparar o impacto ambiental potencial de uma capa de laptop fabricada com materiais reaproveitados pela FAS, com duas outras capas de laptop comerciais (Corino e Neoprene), através da utilização da metodologia de ACV.

\begin{tabular}{|c|c|c|c|c|c|c|c|}
\hline Capa & \multicolumn{2}{|c|}{ Material } & Processo & Componente & $\begin{array}{l}\text { Nomenclatura } \\
\text { SimaPro }\end{array}$ & $\begin{array}{l}\text { Massa } \\
\text { (g) }\end{array}$ & $\begin{array}{r}{ }^{*} \mathrm{MT} \\
(\mathrm{g})\end{array}$ \\
\hline \multirow{7}{*}{$\begin{array}{c}\text { Capa } \\
\text { artesanal } \\
\text { produ- } \\
\text { zida por } \\
\text { uma APL } \\
\text { da FAS }\end{array}$} & \multicolumn{2}{|c|}{ Barbante } & $\begin{array}{c}\text { Spinning, bast fibre } \\
\{\text { RoW }\} \mid \text { processing } \\
\mid \text { Conseq, } U\end{array}$ & Algodão & $\begin{array}{c}\text { Cotton fibre }\{\text { RoW }\} \mid \\
\text { cotton produc- } \\
\text { tion | Conseq, } U\end{array}$ & 103,7 & 103,7 \\
\hline & \multirow{3}{*}{ Zíper } & Dentes & $\begin{array}{l}\text { Injection moulding } \\
\{\text { RoW\}| proces- } \\
\text { sing | Conseq, } \mathrm{U}\end{array}$ & Polioximetileno & $\begin{array}{l}\text { Polyoxymethylene } \\
\text { (POM)/EU-27p }\end{array}$ & 12,0 & \multirow{3}{*}{25,5} \\
\hline & & $\begin{array}{l}\text { Fita de } \\
\text { tecido }\end{array}$ & $\begin{array}{c}\text { Weaving, bast fibre } \\
\text { \{RoW\}| processing } \\
\mid \text { Conseq, } U\end{array}$ & Poliéster & $\begin{array}{l}\text { Polyester resin, unsa- } \\
\text { turated }\{\text { RoW }\} \mid \text { pro- } \\
\text { duction } \mid \text { Conseq, } U\end{array}$ & 9,7 & \\
\hline & & Puxador & $\begin{array}{c}\text { Casting, brass } \\
\{\text { RoW }\} \mid \text { proces- } \\
\text { sing } \mid \text { Conseq, } U\end{array}$ & Zinco & $\begin{array}{l}\text { Zinc }\{G L O\} \mid \text { primary } \\
\text { production from con- } \\
\text { centrate } \mid \text { Conseq, } U\end{array}$ & 3,7 & \\
\hline & \multicolumn{2}{|c|}{ Fio de costura } & $\begin{array}{c}\text { Spinning, bast fibre } \\
\{\text { RoW }\} \mid \text { processing } \\
\mid \text { Conseq, } U\end{array}$ & Poliéster & $\begin{array}{l}\text { Polyester resin, unsa- } \\
\text { turated }\{\text { RoW }\} \mid \text { pro- } \\
\text { duction | Conseq, } U\end{array}$ & 0,1 & 0,1 \\
\hline & \multirow{2}{*}{\multicolumn{2}{|c|}{ Malha }} & \multirow{2}{*}{$\begin{array}{c}\text { Tecelagem, Weaving, } \\
\text { bast fibre }\{\text { RoW\} } \mid \text { pro- } \\
\text { cessing | Conseq, U }\end{array}$} & Viscose & $\begin{array}{c}\text { Viscose fibre }\{G L O\} \mid \\
\text { viscose produc- } \\
\text { tion | Conseq, U }\end{array}$ & 124,1 & \multirow[t]{2}{*}{129,3} \\
\hline & & & & Lycra & $\begin{array}{l}\text { Polyol }\{\text { RoW }\} \mid \text { pro- } \\
\text { duction | Conseq, U }\end{array}$ & 5,1 & \\
\hline
\end{tabular}

Tabela 1: Processos e quantidades da capa de laptop artesanal. ${ }^{*}$ MT=massa total. Fonte: os Autores 


\begin{tabular}{|c|c|c|c|c|c|c|c|}
\hline Capa & \multicolumn{2}{|c|}{ Material } & Processo & Componente & Nomenclatura SimaPro & $\begin{array}{c}\text { Massa } \\
(\mathbf{g})\end{array}$ & $\begin{array}{l}{ }^{*} \mathrm{MT} \\
(\mathrm{g})\end{array}$ \\
\hline \multirow{6}{*}{$\begin{array}{c}\text { Capa } \\
\text { Neoprene }\end{array}$} & \multirow{2}{*}{\multicolumn{2}{|c|}{ Neoprene }} & \multirow[b]{2}{*}{-} & \multirow[b]{2}{*}{ Cloroprene } & $3,4 \mathrm{DCB}$ de 1,4 & 122 & \\
\hline & & & & & Sodium hydroxide & & 200 \\
\hline & \multirow{3}{*}{ Zíper } & Dentes & $\begin{array}{c}\text { Injection moulding } \\
\{\text { RoW }\} \mid \text { proces- } \\
\text { sing } \mid \text { Conseq, U }\end{array}$ & Polioximetileno & $\begin{array}{l}\text { Polyoxymethylene } \\
\text { (POM)/EU-27 }\end{array}$ & 12,0 & 25,5 \\
\hline & & $\begin{array}{l}\text { Fita de } \\
\text { Tecido }\end{array}$ & $\begin{array}{c}\text { Weaving, bast fibre } \\
\{\text { RoW\}| processing } \\
\mid \text { Conseq, U }\end{array}$ & Poliéster & $\begin{array}{l}\text { Polyester resin, unsa- } \\
\text { turated }\{\text { RoW }\} \mid \text { pro- } \\
\text { duction | Conseq, U }\end{array}$ & 9,7 & \\
\hline & & Puxador & $\begin{array}{l}\text { Casting, brass } \\
\{\text { RoW }\} \mid \text { proces- } \\
\text { sing } \mid \text { Conseq, U }\end{array}$ & Zinco & $\begin{array}{l}\text { Zinc }\{G L O\} \mid \text { primary } \\
\text { production from con- } \\
\text { centrate | Conseq, U }\end{array}$ & 3,75 & \\
\hline & \multicolumn{2}{|c|}{ Fio de costura } & $\begin{array}{c}\text { Spinning, bast fibre } \\
\{\text { RoW }\} \mid \text { processing } \\
\mid \text { Conseq, U }\end{array}$ & Poliéster & $\begin{array}{l}\text { Polyester resin, unsa- } \\
\text { turated }\{\text { RoW }\} \mid \text { pro- } \\
\text { duction | Conseq, } U\end{array}$ & 0,1 & 0,1 \\
\hline
\end{tabular}

Tabela 2: Processos e quantidades da capa de laptop neoprene. ${ }^{*} M T=$ massa total.

Fonte: os Autores 


\begin{tabular}{|c|c|c|c|c|c|c|c|}
\hline Capa & \multicolumn{2}{|c|}{ Material } & Processo & Componente & Nomenclatura SimaPro & $\begin{array}{c}\text { Massa } \\
(\mathbf{g})\end{array}$ & $\begin{array}{r}{ }^{*} \mathrm{MT} \\
(\mathrm{g})\end{array}$ \\
\hline \multirow{7}{*}{$\begin{array}{l}\text { Capa } \\
\text { Corino }\end{array}$} & \multirow{2}{*}{\multicolumn{2}{|c|}{ Corin }} & \multirow[t]{2}{*}{ - } & Polivinilclorido & $\begin{array}{l}\text { Polyvinylchloride, emulsion } \\
\text { polymerised \{RoW\}| polyvinyl- } \\
\text { chloride production, emulsion } \\
\text { polymerisation | Conseq, U }\end{array}$ & 101,6 & \multirow[t]{2}{*}{127,0} \\
\hline & & & & Poliéster & $\begin{array}{c}\text { Polyester resin, unsaturated } \\
\{\text { RoW }\} \mid \text { production | Conseq, } U\end{array}$ & 25,4 & \\
\hline & \multirow{3}{*}{ Zíper } & Dentes & $\begin{array}{l}\text { Injection moulding } \\
\{\text { RoW }\} \mid \text { proces- } \\
\text { sing } \mid \text { Conseq, } U\end{array}$ & Polioximetileno & $\begin{array}{l}\text { Polyoxymethylene } \\
\text { (POM)/EU-27 }\end{array}$ & 12,0 & \multirow{3}{*}{25,5} \\
\hline & & $\begin{array}{l}\text { Fita de } \\
\text { Tecido }\end{array}$ & $\begin{array}{c}\text { Weaving, bast fibre } \\
\{\text { RoW\}| proces- } \\
\text { sing } \mid \text { Conseq, } U\end{array}$ & Poliéster & $\begin{array}{c}\text { Polyester resin, unsaturated } \\
\{\text { RoW\}| production | Conseq, } \mathrm{U}\end{array}$ & 9,7 & \\
\hline & & Puxador & $\begin{array}{l}\text { Casting, brass } \\
\{\text { RoW } \mid \text { proces- } \\
\text { sing } \mid \text { Conseq, } U\end{array}$ & Zinco & $\begin{array}{l}\text { Zinc }\{G L O\} \mid \text { primary } \\
\text { production from con- } \\
\text { centrate | Conseq, } U\end{array}$ & 3,7 & \\
\hline & \multicolumn{2}{|c|}{ Fio de costura } & $\begin{array}{c}\text { Spinning, bast } \\
\text { fibre }\{\text { RoW }\} \mid \text { pro- } \\
\text { cessing } \mid \text { Conseq, } U\end{array}$ & Poliéster & $\begin{array}{c}\text { Polyester resin, unsaturated } \\
\{\text { RoW\}| production | Conseq, U }\end{array}$ & 0,1 & 0,1 \\
\hline & \multicolumn{2}{|c|}{ Espuma } & - & Poliuretano & $\begin{array}{c}\text { Polyurethane, flexible foam } \\
\{\text { RoW }\} \mid \text { production | Conseq, } U\end{array}$ & 103,2 & 103,2 \\
\hline
\end{tabular}

Tabela 3: Processos e quantidades da capa de laptop corino. ${ }^{*} M T=$ massa total. Fonte: os Autores

Para realização do inventário do ciclo de vida, todas as quantificações de entradas dos processos unitários das capas de laptop foram retiradas de duas bases de dados, a Ecoinvet v.3 e a Industry data 2.0. Essas bibliotecas estão disponíveis dentro do programa SimaPro, versão 8.0.5. As Tabelas 1, 2 e 3 mostram os materiais, processos e quantidades utilizados para a análise de cada capa.

Para o ICV da capa de laptop artesanal, não foram consideradas as quantidades de lona de banner e de espuma, pois estes materiais foram reaproveitados, sendo obtidos pelas APLs através de doação. Desta forma, como já mencionado em 3.2., foi utilizada a abordagem Cut off, em que os materiais reaproveitados não carregam seus impactos oriundos do ciclo de vida da matéria quando virgem.

Desta forma, através da simulação no SimaPro das três capas de laptop, foi possível obter os impactos ambientais potenciais deles, conforme segue Figura 3.

Comparando-se os materiais das três capas de laptop pôde-se verificar que a capa com maior impacto ambiental foi a capa artesanal, com impacto avaliado em $573 \mu \mathrm{Pt}$, mais que o dobro que a capa de neoprene (225 $\mu \mathrm{Pt})$. A capa de corino ficou em segundo lugar na avaliação, com impacto de $369 \mu \mathrm{Pt}$, como mostra a Figura 3.

A principal categoria de dano em relação ao impacto ambiental na capa artesanal foi a de saúde humana, devido principalmente a maior contribuição do indicador de respiração de compostos inorgânicos, relacionado ao material particulado no ar. Este indicador sofreu bastante influência tanto do material malha como o barbante, presentes na capa artesanal.

Além disso, a utilização de eletricidade nos processos de produção no material barbante e as atividades 
relacionadas a produção da fibra de algodão, como a agricultura, presente como matéria-prima base do barbante também foram considerados processos críticos no impacto ambiental da capa artesanal, não somente para o indicador endpoint de saúde humana, mas também para os indicadores endpoint de qualidade de ecossistema e recursos. Isto ocorreu, pois, tanto a agricultura como o uso de eletricidade produziram alto impacto nas categorias de impacto de Mudanças Climáticas e de Energia Não Renovável.

Nas outras duas capas (Corino e Neoprene), o maior impacto foi na categoria de dano recursos, principalmente devido à matéria-prima gás natural e óleo cru. Isto porque o alto consumo de eletricidade para fabricação dos materiais base destas capas e, principalmente, a extração e processamento do petróleo para a fabricação tanto do neoprene como do poliéster e do PVC, base do corino, fez com que as categorias de impacto mais correlacionadas ao petróleo obtivessem os maiores valores de impacto ambiental potencial.

Por fim, realizando uma análise mais criteriosa dos materiais barbante e malha, componentes da capa artesanal, foi visto que o material que mais interferiu na saúde humana foi a malha, seguido pelo barbante. Entretanto, em relação ao impacto em todas as categorias de dano, o material mais crítico foi o barbante.

Desta forma, foi percebido que a opção de produção de uma capa de laptop através de materiais reaproveitados, como a lona de banner e a espuma, acabou, apesar este reaproveitamento, apresentado um impacto ambiental potencial global maior do que o impacto ambiental potencial das duas capas de laptop comerciais estudadas.
Isto ocorreu devido a estratégia adotada de produção, no momento da concepção do produto capa de laptop, de utilizar o barbante para possibilitar uma trama de costura entre as fitas de lona de banner, e também da utilização da malhar para possibilitar a acomodação interna da espuma. Ou seja, apesar da escolha destes materiais (barbante e malha), o excesso de massa utilizado deles para adequar o design e estética do produto artesanal, de modo a viabilizar a sua comercialização, fez com que ocorresse um carregamento de impacto inicialmente não visível, em primeira análise, que foi da eletricidade e da agricultura, tornando o produto desenhado e proposto pela APL da FAS com um pior desempenho ambiental.

\section{CONSIDERAÇÕES FINAIS}

Este trabalho teve como objetivo principal apresentar os resultados da Avaliação do Ciclo de Vida de uma capa de laptop, comparando três modelos, um deles fabricado com materiais reaproveitados, dentro do programa de APLs, vinculado à FAS, Prefeitura de Curitiba, e outros dois modelos confeccionados com produtos industrializados, comuns no mercado.

Foi realizada uma $\mathrm{ACV}$, na qual foi considerado apenas o aspecto ambiental da sustentabilidade. Após o estudo, concluiu-se que a capa com maior impacto ambiental foi a capa artesanal desenvolvida pela FAS. Ou seja, apesar de possuir alguns materiais reciclados, esta capa não foi mais sustentável ambientalmente do que os modelos estudados presentes no mercado. Isso se deve principalmente ao uso de alguns materiais (barbante e malha) que geraram impacto maior que outros materiais, presentes nas capas industrializadas. Preliminarmente, seria possível imaginar que o produto artesanal geraria menor impacto

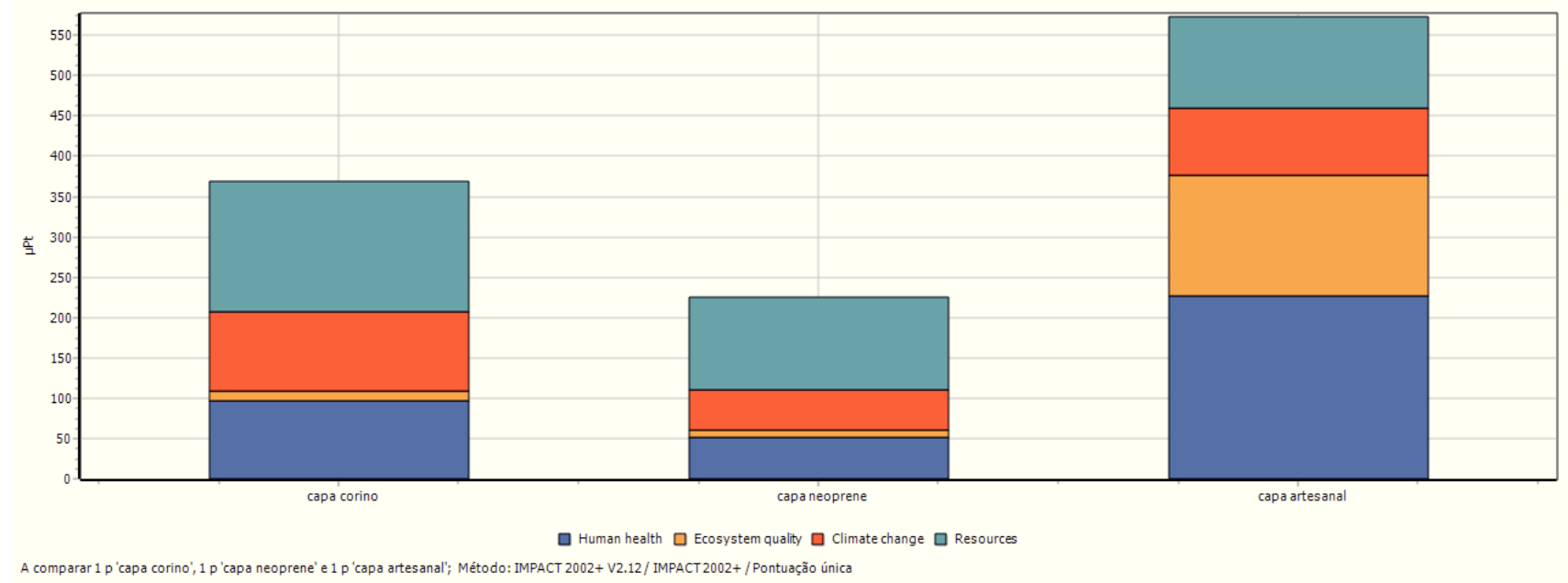

Figura 3: Impacto ambiental capa de laptop.

Fonte: os Autores 
ambiental, entretanto, uma análise criteriosa demonstrou que as capas industrializadas foram mais sustentáveis sob o ponto de vista ambiental.

Apesar disso, pode-se ressaltar que o uso da ACV foi limitado a banco de dados com bases de dados internacionais, com principais contribuições do continente Europeu. Esta limitação pode resultar em deformações dos resultados, necessitando de maiores investigações, de modo a buscar uma maior regionalização deles. Mesmo assim, dados como os de eletricidade foram modificados e regionalizados para o cenário brasileiro.

Outra possível abordagem envolveria uma forma diferente de contabilização dos materiais reaproveitados, por exemplo, considerando a retirada do destino deles como um impacto positivo, a ser descontado do impacto global do produto.

Desta forma, percebe-se que existem ainda alguns aprofundamentos que podem ser realizados para uma abordagem mais ampla e assertiva de produtos, principalmente aqueles com reaproveitamento de materiais. Neste sentido, este estudo abre um leque de possibilidades para futuras pesquisas de ACV no desenvolvimento de novos produtos com base de reaproveitamento.

\section{REFERÊNCIAS}

4LIFESTORE. 2018. Disponível em: http://www.4lifestore.com.br/capa-para-notebook-e-macbook-14-tucano-colore-neoprene-preta-pr-500-395310.htm

Acesso em: 15/08/2018

ADISSI, J.P., et. al. Gestão Ambiental de Unidades

Produtivas. $1^{\text {a }}$ ed. Rio de Janeiro: Elsevier, 2013.

ARRUDA, L. A.; QUELHAS, O. L. G. Sustentabilidade: um longo processo histórico de reavaliação crítica da relação existente entre a sociedade e o meio ambiente. B. Téc. Senac: a R. Educ. Prof., Rio de Janeiro, v. 36, n.3, 2010.

ASSIS, B. B. Avaliação do ciclo de vida do produto como ferramenta para o desenvolvimento sustentável. 53 f. Trabalho de Conclusão de Curso - Universidade Federal de Juiz de Fora, Juiz de Fora, 2009

ASSOCIAÇÃO BRASILEIRA DE NORMAS TÉCNICAS. NBR ISO 14040. Gestão Ambiental - Avaliação do Ciclo de Vida - Princípios e estrutura. Rio de Janeiro, 2009.
ASSOCIAÇÃO BRASILEIRA DE NORMAS TÉCNICAS. NBR ISO 14048. Gestão Ambiental - Avaliação do Ciclo de Vida - Formatos para documentação de dados. Rio de Janeiro, 2002.

CARVALHO, A., MIMOSO, A.F., MENDES, A.N., MATOS, $H$.A. From a literature review to a framework for environmental process impact assessment index. Journal of Cleaner Production, v. 64, p. 36-62, 2014.

CASENOTEBOOK.2018. Disponível em: https://www. casenotebook.com.br/loja/produtos-loja/capa-para-notebook-courino/. Acessado em: 15/08/2018.

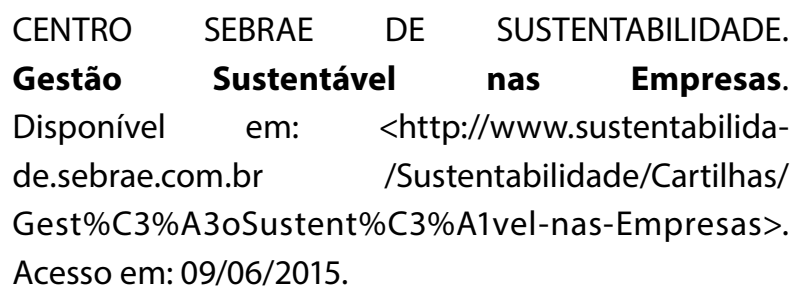

COMISSÃO MUNDIAL SOBRE MEIO AMBIENTE E DESENVOLVIMENTO - CMMAD. Nosso Futuro Comum. Rio de Janeiro: Fundação Getúlio Vargas, 1988

ELKINGTON, J. Triple bottom line revolution: reporting for the third millennium. Australian CPA, v. 69, p. 75, 1994.

FARIA, A. F.; PINTO, C. A.; RIBEIRO, N.M.; CARDOSO T. S.; RIBEIRO, J. P. C. Processo De Desenvolvimento De Novos Produtos: Uma Experiência Didática. In: XXVIII Encontro Nacional de Engenharia de Produção, Rio de Janeiro, 2008. Anais... Rio de Janeiro: ABEPRO, 2008, p. $1-14$

FREITAS, F.L.;FERREIRA, M.P.;MATSUO, T.K.;FORCELLINI, F.A.; Rodrigues, M. A. Processo de desenvolvimento de produto: aplicação em um projeto de P\&D dentro do programa ANEEL. In: XXIV Seminário Nacional de Parques Tecnológicos e Incubadoras de Empresas, Belém, 2014. Anais... Belém: ANPROTEC, 2014, p. 1-16

GMELIN, H.; SEURING, S. Determinants of a sustainable new product development. Journal of Cleaner Production. v. 69, n. 1, p. 1-9, 2014. 
GUTBERLET, J. Cooperative urban mining in Brazil: Collective practices in selective household waste collection and recycling. Waste Management. v. 45, p. 2231, 2015.

HERZOG, A. É politicamente correto e dá mais dinheiro. Exame, São Paulo, p.14-17, dez. 2004a. Edição especial.

JENSEN, A.A., REMMEN, A. Background Report for a UNEP Guide to Life Cycle Management. 2006. Disponível em: www.uneptie.org/pc/sustain/lcinitiative/home.htm, Acessado em: 10/12/2016.

MACENO, M.C.M. EPIP - Ferramenta analítica de desempenho ambiental em processos industriais: desenvolvimento e aplicações. $211 \mathrm{f}$. Tese (Doutorado em Engenharia de Recursos Hídricos e Ambiental) Universidade Federal do Paraná, Curitiba, 2015.

MANZINI, E.; VEZZOLI, C. O desenvolvimento de produtos sustentáveis: os requisitos ambientais dos produtos industriais. São Paulo: Editora da Universidade de São Paulo, 2002.

MIKHAILOVA, I. Sustentabilidade: Evolução dos Conceitos Teóricos e os Problemas da Mensuração Prática. Revista Economia e Desenvolvimento. n. 16, 2004.

OLIVEIRA, L.R.; MEDEIROS, R. M.; TERRAC, P. B.; QUELHAS, O.L.G. Sustentabilidade: da evolução dos conceitos à implementação como estratégia nas organizações. Produção, v. 22, n. 1, p. 70-82, 2012

ROZENFELD, H. et al. Gestão de Desenvolvimento de Produtos: uma referência para a melhoria do processo. São Paulo: Saraiva, 2006.

SIMAPRO Software. Versão 8.0.5. Amersfoort: Pré Consultants, 2015.

UNEP/SETAC. Guidelines for Social Life Cycle Assessment of Products. 2009. Disponível em: http:// Icinitiative.unep.fr. Acesso em: 22/02/2016.

\section{AUTORES}

ORCID: https://orcid.org/0000-0003-1291-7177

LUISA PEREIRA BASILE. | Universidade Federal do Paraná | Centro Politécnico | Engenharia de Produção | Curitiba, PR. Brasil | Correspondência para: Rua Francisco H. dos Santos, n. 210 - Centro Politécnico / Setor de Tecnologia Bairro: Jardim das Américas - Curitiba-PR - CEP: 81531-980 - Caixa Postal: 19011 | E-mail: luisa.p.basile@gmail.com

ORCID: https://orcid.org/0000-0002-6762-8236

MARCELL MARIANO CORRÊA MACENO, DR. | Universidade Federal do Paraná | Centro Politécnico | Engenharia Ambiental | Curitiba, PR. Brasil | Correspondência para: Rua Francisco H. dos Santos, no. 210 - Centro Politécnico / Setor de Tecnologia - Bairro: Jardim das Américas - Curitiba-PR CEP: 81531-980 - Caixa Postal: 19011 | E-mail: marcell.maceno@gmail.com

ORCID: https://orcid.org/0000-0001-7240-1143

ADRIANA DE PAULA LACERDA SANTOS, DRA. | Universidade Federal do Paraná | Centro Politécnico | Engenharia de Produção | Curitiba, PR. Brasil | Correspondência para: Rua Francisco H. dos Santos, n. 210 - Centro Politécnico / Setor de Tecnologia - Bairro: Jardim das Américas - Curitiba-PR - CEP: 81531-980 - Caixa Postal: 19011 | E-mail: lacerda@ gmail.com

\section{COMO CITAR ESTE ARTIGO}

BASILE, Luisa Pereira; MACENO, Marcell Mariano Corrêa; SANTOS, Adriana de Paula Lacerda. Comparação do Impacto Ambiental de Capa de Laptop Utilizando a Avaliação do Ciclo de Vida. MIX Sustentável, [S.I.], v. 4, n. 3, p. 49-59, out-mar. 2018. ISSN 24473073. Disponível em: <http://www.nexos.ufsc.br/index.php/ mixsustentavel>. Acesso em: dia mês. ano. doi:https:// doi.org/10.29183/2447-3073.MIX2018.v4.n3.49-59.

DATA DE ENVI0: 04/09/2018

DATA DE ACEITE: 10/09/2018 